\title{
Éxitos y fracasos de la cooperación transfronteriza: El ejemplo del Bidasoa
}

\section{Successes and failures of cross-border collaboration: The example of the Bidasoa}

Juan Cruz Alberdi Collantes ${ }^{\mathrm{a}^{*}}$ (D) https://orcid.org/0000-0002-1109-4925
Recibido el 6 de septiembre de 2019. Aceptado el 16 de diciembre de 2019. Publicado el 20 de diciembre de 2019.

*Autor para correspondencia: Juan Cruz Alberdi Collantes, correo electrónico, juancruz.alberdi@ehu.eus

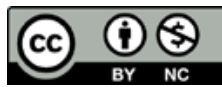

Esta obra está protegida bajo una Licencia Creative Commons Atribución-NoComercial 4.0 Internacional.

\begin{abstract}
${ }^{a}$ Universidad del País Vasco, Departamento de Geografía, Facultad de Filosofía y Geografía e Historia, España, correo electrónico: juancruz.alberdi@ehu.eus
\end{abstract}

\section{Resumen:}

La comarca del Bajo Bidasoa, que engloba a los municipios de Irun y Hondarrabia en España y a Hendaya en Francia, lleva décadas cultivando la colaboración intermunicipal, especialmente tras la puesta en marcha del consorcio transfronterizo Bidasoa-Txingudi el año 1998. La investigación tiene el objeto de mostrar la actividad de esta entidad como ejemplo de desarrollo de las políticas entre límites fronterizos interestatales dentro de la Unión Europea, y de los esquemas de socialización espacial que operan en torno a ellas. Optamos por la entrevista en profundidad para contrastar los resultados obtenidos mediante fuentes publicadas. Los resultados dejan entrever una incuestionable voluntad de materializar una colaboración limitada, sin embargo, por la dificultad de iniciar proyectos comunes regidos por legislación de dos Estados diferentes.

Palabras clave: conurbación municipal, agencias de desarrollo, Bidasoa, País Vasco, colaboración transfronteriza, frontera.

\begin{abstract}
:
The Lower Bidasoa region, which encompasses the municipalities of Irun and Hondarrabia in Spain and Hendaye in France, has been cultivating municipality collaboration for decades, especially after the foundation of the international partnership of Bidasoa-Txingudi in 1998. The objective of the investigation is to show the activity of this entity as an example of the development of policies between interstate border boundaries in the European Union, and of the spatial socialization schemes that operate around them. We opted for the in-depth interview to contrast the results obtained through published sources. The results suggest an unquestionable willingness to materialize this relationship. However, this collaboration is limited by the difficulty of initiating common projects governed by legislation from two different States.
\end{abstract}

CÓMO CITAR: Alberdi, J. C. (2019). Éxitos y fracasos de la cooperación transfronteriza: El ejemplo del Bidasoa. [Successes and failures of cross-border collaboration: The example of the Bidasoa]. Estudios Fronterizos, 20, e041. doi:https://doi.org/10.21670/ ref.1920041 
Keywords: conurbation, development agency, Bidasoa, Basque Country, international partnership, border.

\section{Introducción al objeto de la investigación}

Durante el curso de los últimos decenios, la aceleración del proceso de globalización de los intercambios económicos y culturales, la recomposición de la territorialidad de los Estados y el fortalecimiento de los mecanismos de integración regional han conducido a una mutación de las funciones tradicionalmente atribuidas a las fronteras estatales (Anderson y O'Dowd, 1999). De barrera territorial que desempeña un papel estructurante en la diferenciación de los sistemas políticos y económicos, pero también en la afirmación de culturas y de identidades distintas, la frontera aparece en lo sucesivo como una interfaz que favorece los contactos y los intercambios (O'Dowd, 2010). Ahora, el desarrollo de zonas urbanas funcionales y discontinuas ya no se restringe a los límites de los territorios nacionales, sino que se concierne de más en más a los espacios fronterizos (Sohn, Reitel y Walther, 2009).

La desaparición de las fronteras estatales supone un paso fundamental en la liberalización de las transacciones económicas y en la consecución de un mercado común que, de manera progresiva, nos conduce a otro escenario económico-social. Se suceden iniciativas europeas, nacionales y regionales, pero son las instituciones locales, los ayuntamientos, los que comparten, producto de la proximidad física, una relación diaria que se materializa a ambos lados de la frontera (Barrios, 2013).

En este trabajo, el acercamiento a la frontera se produce desde su comprensión como un proceso inacabado. Si las fronteras crecieron como parte de los Estados, las identificaciones políticas se construyen a través de prácticas de fronterización (Kuus, 2010), entendidas como una amplia gama de procesos transformativos y afectivos en los cuales los órdenes y desórdenes sociales y espaciales son constantemente reelaborados (Woodward y Jones, 2005). Repensar las fronteras a través de las prácticas de fronterización supone entender esas prácticas como algo implícito en la construcción de las mismas, no analizables como desarrollos incompletos o acabados, sino en constante proceso de materialización (Prokkola, 2008).

El objetivo de este artículo es presentar el consorcio transfronterizo del Bidasoa como ejemplo de desarrollo de las políticas entre límites fronterizos interestatales dentro de la Unión Europea (UE), un modelo con avances interesantes pero con barreras que tras décadas ha sido incapaz de franquear y que limitan sus opciones de progreso de futuro

El espacio elegido conforma una unidad geográfica claramente delimitada, con unos atributos naturales, sociales y económicos comunes y confluyentes, y con una dinámica de relación materializada en instituciones comunes con una trayectoria de varias décadas (Arbaiza, 2006). La Comarca del Bajo Bidasoa constituye un laboratorio ideal para observar el avance de la colaboración entre comunidades locales fronterizas, especialmente a partir de la supresión física de la frontera. En él confluye el interés de las instituciones por potenciar una gobernanza común, la necesidad de ampliar la actividad económica hacia nuevos mercados de proximidad e incluso la propia idiosincrasia vasca, con una cultura e idioma común dividido en dos territorios fronterizos. 
La investigación retoma contribuciones previas (Alberdi, 2006; 2013) centradas en esta problemática y en este entorno, renovándolas con nuevas aportaciones centradas en otros ejemplos prácticos, actualiza la situación que atraviesa la cooperación local entre Francia y España en la última década y analiza la incidencia de nuevas entidades de cooperación transnacional que actúan a escala local.

La metodología utilizada se fundamenta en la recogida de información publicada, de origen muy diverso, puesto que el área no ha sido objeto de ningún estudio pormenorizado (informes anuales, hemeroteca, estudios de casos, entre otros). Pero estas fuentes requieren ser complementados con la información aportada por otros métodos. En esta investigación apostamos por la entrevista en profundidad como técnica capaz de ofrecer el contraste cualitativo a los resultados obtenidos mediante fuentes publicadas, y facilitar su posterior comprensión (Guerrero, 2001).

Dos aspectos son relevantes en la preparación de las entrevistas en profundidad de esta investigación. En primer lugar, la planificación del guión de la entrevista, que contiene los temas que deben cubrirse, que varían según la responsabilidad del entrevistado, pero que se centran principalmente en valorar el recorrido de la entidad de cooperación. Elegimos un esquema abierto, con puntos a tratar, cuyo orden no tiene que seguirse necesariamente, que recoge el flujo particular de información del entrevistado y que capta aspectos no previstos en el guion. En segundo lugar, la selección de entrevistados. Optamos por entrevistas dirigidas a los agentes técnicos y políticos con responsabilidad en la promoción de acciones transfronterizas, entablando conversaciones con aquellos que lideran tanto proyectos específicos como la gestión y responsabilidad del consorcio.

Se entrevistó a los dos técnicos del consorcio, a los encargados de Bidasoa Activa responsable de la promoción turística del área y de los proyectos liderados en colaboración con la Communauté Sud Pays-Basque y a un representante de cada uno de los ayuntamientos en el Consorcio Transfronterizo. Las entrevistas se realizaron entre octubre del año 2016 y marzo de 2017. En el marco de un proyecto de investigación "La cooperación transfronteriza en Europa. Una geopolítica a escala local".

\section{Estado de la cuestión: un grado de colaboración desigual y diferente según escala y región}

Desde los años noventa, la literatura sobre política europea ha aumentado su atención en las regiones transfronterizas. Académicos como Scott (2007), Perkmann y Sum (2002), entre otros, han presentado estudios sobre los cambios contemporáneos en las regiones transfronterizas. Estos autores han tratado de crear un paradigma que explique por qué ocurre la integración transfronteriza y cómo este proceso ha afectado a sus comunidades.

La mayor parte de estos estudios examinan las medidas específicas de política europea que han desarrollado la integración fronteriza en el continente. Las primeras de estas medidas se recogen en los Acuerdos de Schengen, firmados en 1985 y ampliados en 1990, los cuales abolieron los controles de las fronteras entre la mayoría de los Estados miembros de la UE. Como apunta Koff (2008), gran parte de los académicos coinciden en que estos acuerdos crearon dos clases de fronteras en la UE: una interna y otra externa. 
Por ejemplo, Luxemburgo ha formado La Gran Región con partes de Alemania, Francia y Bélgica. Esta fusión ha ayudado a Luxemburgo a transformarse de un pequeño Estado-nación a una capital regional. Investigadores que han estudiado La Gran Región indican que más de 120.000 personas cruzan las fronteras nacionales diariamente por razones profesionales (Berger, 2005; Sohn et al., 2009; Statec, 2007).

LosAcuerdos de Schengen también han tenido el efecto contrario en las comunidades situadas en las fronteras externas de la UE. Esto ha afectado especialmente a Europa del Este. Investigadores como Alexeev (1999) han afirmado que la implementación de los Acuerdos de Schengen dividió artificialmente las economías fronterizas que habían sido alimentadas por la colaboración intergubernamental. Algunos estudiosos, como Kupiszewski (2004), se refieren a las fronteras Schengen como "la nueva cortina de hierro".

Profundizando en las fronteras interiores, otra de las contribuciones importantes de la UE, para impulsar la integración transfronteriza, ha sido la creación de políticas económicas de desarrollo que se centran en regiones geográficas y económicas antes que en Estados. Los programas que reciben la denominación de transfronterizos se han multiplicado desde el nacimiento de Interreg, a principios de los años noventa. La mayor parte de la investigación sobre fronteras europeas ha establecido acertadamente que su más grande contribución ha estado en el campo de la gobernanza. Los programas Interreg han creado comunidades trasnacionales dentro de la uE y han reforzado las fronteras externas de la unión, por lo cual sostienen, además, que la integración fronteriza mejora la cohesión social en comunidades afectadas (VenturaFernández, 2013).

Otros analistas, sin embargo, profundizan en la integración que gracias a estas políticas se ha producido. Koff (2008), a partir del estudio de diferentes ejemplos, señala que en la integración fronteriza los actores locales son quienes determinan el carácter real de tales procesos, y distingue dos realidades diferenciadas, especialmente en el análisis de la colaboración regional: aquellas en las que la integración se realiza exclusivamente al nivel de las élites, porque hay pocos estímulos para compartir sus beneficios económicos con el conjunto de la población; y, por el contrario, en áreas donde no hay actores dominantes, los beneficios de integración se dispersan y la cohesión social mejora. Sostiene que las estructuras locales de poder explican la integración fronteriza más efectivamente que las aproximaciones institucionalistas que dominan actualmente la literatura sobre la política fronteriza en Europa

Descendiendo en la escala de colaboración, la proximidad física implica de un modo $\mathrm{u}$ otro a todo el tejido social, las relaciones se incrementan y la reciprocidad avanza de forma visible. Son muchos los estudios que analizan esta relación (Prokkola, 2008; Matthiessen, 2005, Rogut y Welter, 2012). Centrándonos en los casos de colaboración relacionados con España (Oliveras, 2013; Barrios, 2013; Feliu, Berzi, Rufí, Castañer y Llussà, 2013; Lois y Carballo, 2015; Márquez, 2016; Martín-Uceda y Castañer, 2018), parecen observarse varias tendencias que bien merecen una reflexión.

- Un primer elemento a destacar es la consolidación de los espacios de cooperación.

Estas estructuras demuestran, en primer lugar, como los actores regionales y locales, que habían tenido un papel secundario en las relaciones transfronterizas, a lo largo de los años después del cambio de significado de las fronteras, transforman 
su rol y empiezan a tomar parte en este proceso. Se refuerza su capacidad para emerger como actores conjuntamente al Estado central.

- El desarrollo económico es la temática principal de la mayoría de los proyectos aprobados e impulsados. Este hecho supone la constatación que los espacios fronterizos tienen, en la mayoría de los casos, todavía dificultades para impulsar estrategias económicas que permitan el crecimiento económico sostenible en el tiempo, al mismo tiempo que este tenga un impacto positivo en las estructuras socioeconómicas del territorio local.

- En cuanto a las motivaciones que llevan a impulsar la institucionalización de la relación transfronteriza, a menudo se ha reducido a un simple interés de obtención de fondos europeos de los programas Interreg. Según esta argumentación, de no ser por la posible financiación exterior, no se habrían llevado a cabo muchos de los proyectos. Sin embargo, los investigadores muestran que también existen otras motivaciones, siendo en ocasiones más relevantes. Pueden nombrarse la resolución de conflictos fronterizos, la agregación de valor añadido en sectores económicos en crisis y con necesidad de innovaciones y nuevos mercados, o la formación de cuencas de población suficientes para la planificación de servicios, equipamientos o infraestructuras de escala supramunicipal (cooperación en sanidad y en enseñanza).

- Respecto al objeto de la cooperación, las investigaciones establecen una doble diferenciación. Por un lado, la cooperación respecto a los servicios, equipamientos... ya existentes o nuevos, que son redimensionados en el marco de un nuevo espacio transfronterizo, representada, entre otros, por los convenios para la atención sanitaria; la gestión compartida de elementos naturales; o la urbanización de espacios interurbanos. Por otro, la puesta en marcha de iniciativas que potencien la promoción conjunta de la zona con la idea de cohesionarla internamente (actividades escolares, actos festivos...) y como imagen común respecto al exterior, con iniciativas ligadas a la promoción turística.

La evaluación de los procesos y los resultados presenta valoraciones distintas en cada caso, no generalizables al conjunto. La cooperación en sanidad, construcción y puesta en marcha del Hospital de Cerdanya (Oliveras, 2013), puede calificarse de éxito. Al contrario, la cooperación en enseñanza parece haber fracasado en aquellos casos que implican la construcción y puesta en práctica de centros educativos compartidos, pero han supuesto un éxito los intercambios y fiestas escolares y la elaboración de materiales pedagógicos (Feliu et al., 2013).

El ritmo también parece variar en función de la situación socioeconómica del momento, apuntando algunos investigadores que la cooperación se fortalece en contextos expansivos mientras acusa problemas en un marco de empobrecimiento de las sociedades fronterizas, como ha quedado demostrado en la crisis económica de principios de la década, con un descenso acusado del movimiento de trabajadores, turistas e incluso comercio entre ambos lados de las regiones fronterizas (Lois y Carballo, 2015).

En todo caso, las razones del éxito o fracaso pueden ser varias, de las que pueden destacarse las siguientes: desavenencias entre los agentes, ya sea entre los dos lados o de los mismos; ritmos administrativos, políticos, técnicos o financieros lentos o incompatibilidades, en contraposición a las expectativas creadas; o permanencia de las barreras (psicológicas, lingüísticas, legales, etc.). 
Las investigaciones coinciden en que, a pesar de todos los esfuerzos, la utilidad de la colaboración es menor a la deseada. Resulta sorprendente la ausencia de una mayor coordinación y reflexión global e integral a escala comarcal, evaluación de la coherencia de los proyectos o puesta en común de los resultados y aprendizajes. La falta de coordinación también resulta patente en un espacio transfronterizo mayor, entre los organismos de cooperación de escala regional que asumen en menor o mayor grado esta función (Oliveras-González, Colomb y Durà-Guimerà, 2016).

\section{Aproximación al marco territorial: De la cooperación transpirenaica a la conurbación del Bidasoa}

A pesar del considerable papel que la frontera política ha ejercido como barrera y tamiz, flujos y relaciones entre un lado y otro de esta frontera se han sucedido a lo largo del tiempo (Fernández, 1985). En la frontera España-Francia el primer paso de cooperación institucional, sin embargo, no se produce hasta el año 1983, con la fundación de la Comunidad de Trabajo de los Pirineos (СтP). Desde entonces se han establecido varios organismos y proyectos de cooperación, desde la escala regional hasta la local. ${ }^{1}$

La plena integración en el marco institucional europeo es la clave para entender la implantación de la moderna cooperación transfronteriza. Según Oliveras (2013), son tres los factores condicionantes. En primer lugar, la descentralización de España y Francia se acompañó de la dotación de autonomía política y financiera a sus entidades subestatales, lo que facilitó el establecimiento de acuerdos de cooperación transfronteriza, aunque no fue hasta 1992, en el caso francés, y 1997 en el español, cuando los Estados legislaron en este sentido. Ambas legislaciones son similares: obligan a una comunicación previa a la administración central, una restricción dentro de los límites competenciales y el respeto a los compromisos internacionales del Estado. Asimismo, definen la personalidad jurídica de los organismos de cooperación (Agrupación de Interés Público, Distritos Europeos y Consorcios).

En segundo lugar, en 1980, El Consejo de Europa aprobó el Convenio-marco europeo de cooperación transfronteriza entre autoridades y comunidades territoriales (la primera regulación jurídica de sus características). España y Francia lo ratificaron en 1990, seguido en 1995 de la rúbrica de un acuerdo bilateral, el Tratado relativo a la cooperación transfronteriza entre colectividades territoriales (o Tratado de Bayona). Por este tratado se autoriza a las respectivas entidades subestatales fronterizas a establecer convenios de cooperación y constituir organismos con los objetivos de crear y gestionar equipamientos y servicios públicos, estudiar y coordinar decisiones en los dominios de interés común y formular propuestas de cooperación. En 2006 la UE creó su propia regulación jurídica, el reglamento sobre las Agrupaciones Europeas de Cooperación Territorial (AECT). Tres años después España y Francia acordaron el procedimiento de participación de sus entidades subestatales.

\footnotetext{
${ }^{1}$ Son varias las investigaciones que analizan la evolución de la cooperación transfronteriza en la frontera pirenaica, algunas materializadas hace ya tres décadas. En esta investigación resaltamos las aportaciones de Altemir (1989), Llimona I Balcells (2001), Arbaiza (2006) y Oliveras (2013), cuyas aportaciones en buena medida seguimos en esta investigación.
} 
En tercer y último lugar, en 1989, la UE puso en marcha los programas Interreg para la financiación de proyectos de cooperación transfronteriza. ${ }^{2}$ Para la frontera hispanofrancesa se ha canalizado mediante el programa operacional Interreg FranciaEspaña Pirineos y, a partir de la reforma de 2007, Cooperación Transfronteriza EspañaFrancia-Andorra (POCTEFA). En los primeros años su gestión fue monopolizada por los Estados. Solo tardíamente se integraron las entidades subestatales, designándose en 2007 a la CTP como autoridad de gestión (Heredero y Olmedillas, 2009; Harguindéguy y Rodríguez, 2010, Feliu et al., 2013).

Ante estos condicionamientos la Comunidad Autónoma del País Vasco ha incorporado o promovido una serie de asociaciones o instituciones de cooperación internacional (Galdos y Ruiz, 1995), algunas de ámbito más extenso, las denominadas macro asociaciones, y otras más ligadas a la frontera entre España y Francia y al ámbito vasco (Ibarra e Ikardo, 1997).

El Protocolo de Cooperación Euskadi-Aquitania nace para el intercambio de información sobre sus respectivas políticas en diferentes ámbitos como el económico y el social, para impulsar y promover la formación y la investigación, así como la revalorización del patrimonio cultural y lingüístico (Fourny y Velásco-Graciet, 2005). En este sentido desde hace dos décadas se habilita un Fondo Común dentro del Protocolo, financiado a partes iguales entre la Comunidad Autónoma del País Vasco y el Consejo Regional de Aquitania, para la financiación de diferentes proyectos.

Esta última década se da un nuevo impulso en este ámbito de relación regional, con la creación de la Euroregión Euskadi-Aquitania en diciembre de 2011 y su ampliación a Navarra a partir del año $2017,{ }^{3}$ cuya sede principal se instala en Hendaya. Mejorar las comunicaciones de transporte parece ser, hasta el momento, el objetivo prioritario de esta nueva entidad con tres áreas principales de trabajo, la alta velocidad, el transporte interno, y el transporte marítimo.

Parece evidente que desde la uE se está apostando por desarrollar proyectos de infraestructuras interregionales y en su ejecución las euroregiones adquieren un papel estratégico. En todos estos ámbitos se están dando pasos pero al menos hasta el momento, no han superado la fase de proyección y apenas se han materializado acciones en las áreas de trabajo propuestas.

Respecto al convenio entre la Diputación de Gipuzkoa y el Distrito Biarritz-AngletBayona, la realidad física y social del área urbana Bayona-Donostia ha determinado la necesidad de una cooperación por encima de las fronteras que logre estructurar y desarrollar dicho espacio urbano. Se pretende potenciar el sistema de ciudades medias en la zona atlántica frente a los espacios europeos centrales y del Mediterráneo. La eurociudad vasca, según Sansinenea (2001), es uno de los nodos del Arco Atlántico en proyecto, de cara a competir o colaborar con las metrópolis europeas más prósperas, que además juega unas bazas propias de una región fronteriza, que le otorgan su carácter distintivo. Pero, hasta el momento, la colaboración en la eurociudad, tal y

\footnotetext{
${ }^{2}$ La puesta en marcha de instrumentos europeos de financiación se justifica, como sugiere Llimona I Balcells (2001), porque el proceso de integración europea confiere a las regiones que han sido divididas por una frontera, la oportunidad y en cierto sentido también la obligación de recuperar y revitalizar los vínculos entre ellas. Además, la cooperación transfronteriza constituye también un elemento enormemente valioso para el acercamiento entre los pueblos europeos.

${ }^{3}$ Consultar en: https://www.euroregion-naen.eu/leuroregion/historique/
} 
como ocurre en la Euroregión, tan solo se ha establecido a nivel de proyectos que excepcionalmente se han materializado. ${ }^{4}$

Es en el ámbito local, sin embargo, donde los proyectos sí se están materializando, especialmente en la conurbación del Bidasoa. La comarca recibe el nombre de Bidasoa-Txingudi en referencia a dos accidentes geográficos fundamentales en su configuración como es río Bidasoa y la bahía de Txingudi. Este río nace en los montes de Navarra y desemboca en la bahía de Txingudi marcando el límite natural entre España y Francia. Está constituida por los municipios de Irun y Hondarribia, administrativamente en la Comunidad Autónoma del País Vasco, y el municipio de Hendaya, dependiente del Departamento de los Pirineos Atlánticos y de la región de la Nueva Aquitania (Figura 1).

\section{Figura 1: Conurbación de la Bahía de Txingudi}

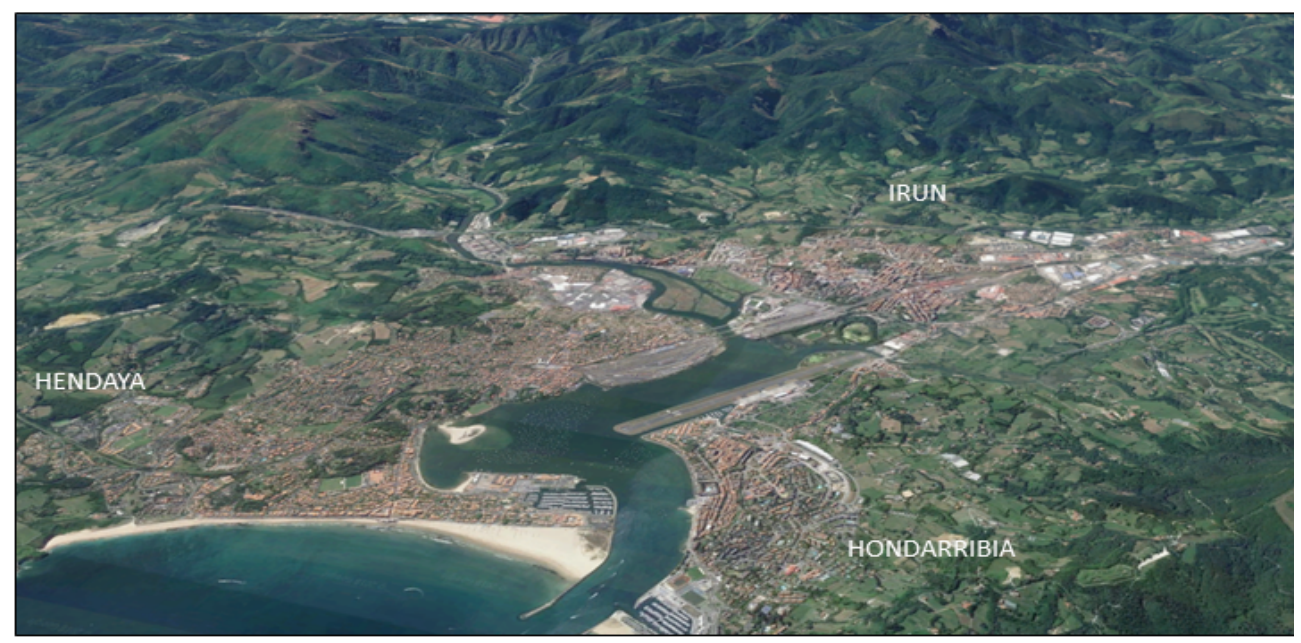

Fuente: Google Earth. Elaboración propia.

La función fronteriza que cumple ha afectado en multitud de aspectos de su configuración social, desde la fundación de sus villas hasta su organización contemporánea. Su impronta actual queda reflejada espacialmente en dos modelos territoriales diferenciados y muy contrastados cuya máxima expresión se evidencia entre Irun y Hendaya. ${ }^{5}$

\footnotetext{
${ }^{4}$ Lozano (2007) llega a la conclusión de que el proyecto de la Eurociudad Baiona-Donostia supuso un gran reto a futuro para superar las fronteras interiores de la UE y desarrollar una región establecida a ambos lados del Bidasoa de forma equilibrada y conjunta. No obstante, los resultados después de una década no parecen ser excesivamente optimistas. Las trabas político-administrativas, la falta de voluntades y de unos fondos amplios ha dado al traste o no ha desarrollado convenientemente todo aquello que fue planificado en un principio.

${ }^{5}$ A raíz de la aprobación del proyecto InnovMugabe Adebisa y la Communauté Sud PaysBasque crean un observatorio en el que se recogen los datos más significativos de la población y de la economía de las localidades que componen esta comarca. Se pueden consultar en la wed del proyecto www.bidasoa-sudpaysbasque.com
} 
Irun, desarrollado principalmente sobre los aluviones originados por el río Bidasoa, concentra la mayoría de las infraestructuras económicas de la comarca y, junto a ello, continúa incrementando su peso poblacional, con 61.239 habitantes en la actualidad. Principal paso de mercancías rodadas de la península Ibérica, saca partido de su ubicación estratégica desarrollando todo tipo de servicios dirigidos a compañías de transporte. Esta reorientación de la actividad aduanera que tradicionalmente ha ejercido el municipio le ha posibilitado, tras al menos una década de incertidumbre, recuperar parte de su pujanza económica anterior.

Hendaya, con algo más de 15000 habitantes, se caracteriza por el dominio de la rama del turismo y del comercio y, en su segundo lugar, el empleo en transporte y comunicaciones. Como principal punto estratégico del municipio cabe señalar la estación y plataforma del ferrocarril. En este lugar se efectúa el trasbordo internacional de personas y mercancías. Junto al transporte cabe destacar la actividad comercial municipal, cuya actividad se ve reforzada en época estival, momento en el cual Hendaya llega a quintuplicar la población residente. Cuenta con 22 hoteles, 7.215 plazas de camping, 270 apartamentos turísticos y alrededor de 5.000 residencias secundarias. Este turismo, sin embargo, se concentra en los meses de verano, siendo la estacionalidad uno de los problemas principales a los que actualmente se enfrenta la villa.

Hondarribia, ubicada en la margen izquierda del río Bidasoa, protegida al oeste con las estribaciones del monte Jaizkibel, desarrolla su casco histórico en un pequeño promontorio rodeado de murallas mientras, tras estas, surge el barrio de la Marina, un poblado típico de pescadores que contrasta con la nobleza del casco medieval. Todo ello lleva a aportar a Hondarribia el título de Monumento Histórico Nacional. En la actualidad el municipio, con 16.500 habitantes, desarrolla su función residencial, atrayendo a población que trabaja en Irun y en San Sebastián mientras las actividades turísticas y recreativas adquieren cada vez más presencia (Tabla 1).

Tabla 1: Empleo por sectores económicos \% (2016)

\begin{tabular}{|c|c|c|c|}
\hline & Hendaya & Hondarribia & Irun \\
\hline $\begin{array}{c}\text { Empleo sector industrial } \\
(\%)\end{array}$ & 12,91 & 3,6 & 18,65 \\
\hline $\begin{array}{c}\text { Empleo sector construcción } \\
(\%)\end{array}$ & 4,71 & 6,2 & 16,6 \\
\hline $\begin{array}{c}\text { Empleo sector servicios } \\
(\%)\end{array}$ & 81,37 & 80,57 & 76,09 \\
\hline
\end{tabular}

Fuente: Bidasoa Activa (2019).

Pero, además de las peculiaridades propias de cada municipio, uno de los aspectos que caracterizan a la comarca y que le aporta el carácter tan especial de su ubicación en una zona limítrofe es, sin duda alguna, el trasvase de población que se está produciendo de un lado a otro de la frontera y que se refleja muy bien en la caracterización actual del municipio de Hendaya. 
El movimiento de población desde Gipuzkoa hacia Hendaya y los municipios colindantes no es nuevo, pero tiende a incrementarse. Actualmente, al menos uno de cada tres vecinos de la localidad vascofrancesa es de procedencia guipuzcoana. Según el Instituto de Estadística de Francia, en torno al 28\% de los 15.000 habitantes actuales es de origen guipuzcoano cuando en 1999 de sus 10.000 habitantes solo lo era el $13 \%$. Otras cuantificaciones elevan actualmente el porcentaje al $48 \%$ de la población, apoyados en estudios de matriculación y en información municipal referente a altas de electricidad e impuestos se llega a alcanzar los datos señalados (Sallaberry, 2012).

\section{Resultados de la investigación}

A partir de la información publicada, disponible en hemeroteca e informes anuales preferente, valorizada con los resultados de las entrevistas semiestructuradas, obtenemos una visión de la situación que atraviesa actualmente la relación institucional transfronteriza entre los municipios del Bidasoa, que recogemos en tres apartados diferenciados; descripción del modelo de cooperación elegido, valorización de los resultados y retos a afrontar.

\section{Consorcio municipal: Figura elegida para la cooperación en el Bidasoa}

El Consorcio transfronterizo entre Hendaya, Irun y Hondarribia (1998) materializa un proyecto jurídico común. Esta entidad además nace con el objeto de fomentar un desarrollo socioeconómico común impulsando actuaciones y estudios que puedan valorizar la comarca. ${ }^{6}$

Siguiendo las aportaciones de Alberdi (2006), la creación del Consorcio está precedida de una colaboración anterior. El primer antecedente de la misma data de 1990, año en el que se firma la Declaración Institucional sobre la comarca del Bajo Bidasoa ante el Mercado Único. Siguiendo las directrices de esta declaración de intenciones se pone en marcha entre otras acciones la elaboración del Plan Estratégico del Bidasoa en 1993, en el que se establecen las líneas de actuación a medio plazo para la dinamización y desarrollo económico de la comarca del Bidasoa. En dicho plan se apuntan acciones como la creación de un Instituto de Transporte, Comercio y Marketing o la proyección de la comarca como único destino turístico. Las directrices tienen la virtud de abrir una línea de trabajo que se materializará en la consecución de un convenio ese mismo año para la constitución del Eurodistrito Bidasoa-Txingudi (Adebisa-Bidasoa Activa, 1993).

Este primer paso institucional se apoya en el convenio Marco Europeo de Cooperación Transfronteriza de las colectividades o autoridades territoriales, que entró en vigor en 1981 y que es ratificado por los Estados español y francés con la

\footnotetext{
${ }^{6}$ Para profundizar en los antecedentes y objetivos del Consorcio consultar su página web: www.bidasoa-txingudi.com. En ella se recoge información sobre su composición, formación, estatutos y se detalla las actividades y líneas principales abordadas desde esta entidad.
} 
aprobación el tratado de Bayona (1995). Establece el contexto legal concreto en el que se basa la experiencia del Consorcio Bidasoa-Txingudi, exigiendo el tratado la conclusión de un convenio entre las entidades territoriales que pretendan crear y gestionar equipamientos o servicios públicos y coordinar sus decisiones en los ámbitos de interés común, que se firma finalmente el 23 de diciembre de 1998. El convenio, en principio, se firma con el objeto de cumplir los fines que de manera resumida a continuación describimos:

- Turismo: Entre otros aspectos se pretende realizar una oferta común de los tres municipios, seguir una política similar, promocionarse de manera conjunta, crear un centro de iniciativas dirigidas desde el mismo Consorcio.

- Cultura: promover el aprendizaje de las diferentes lenguas, realizar cursos de formación cualificante, realización de manifestaciones culturales.

- Social: establecimiento de un servicio común de acogida de colectivos emigrantes con dificultades, puesta en marcha de una oficina de información social, asesoramiento a profesionales y ciudadanos sobre legislación social y sanitaria.

- Desarrollo económico: creación de una célula de información, asesoramiento y de formación para los impulsores de proyectos, organización de transportes regulares fronterizos, favorecer el establecimiento de una plataforma intermodal y participar en su gestión.

Respecto a la figura elegida en la materialización del Consorcio, este nace como una administración pública de carácter extraordinario, consideración que se produce por la necesidad que tiene de cumplir tanto la legislación española como la francesa. En principio, se regula en función de la legislación española pero todas sus iniciativas han de estar avaladas por la administración francesa, al ser un ámbito de actuación supranacional. Ello requiere un trabajo de coordinación interadministrativa muy importante y casi imposible una vez que se supera el ámbito competencial municipal.

En relación con su régimen interno, el Consorcio está gobernado por un Consejo General compuesto por los tres alcaldes junto a dos representantes de cada municipio. En este Consejo cada persona tiene un voto, independientemente del presupuesto que aporte cada municipio (Irun el 50\%). Sin embargo, para que un proyecto pueda ser iniciado necesita al menos el apoyo de dos de los representantes de cada villa, lo que en la práctica lleva a asegurar el consenso.

Si el Consejo toma las decisiones, es el Comité de Dirección el órgano encargado de poner en marcha las iniciativas adoptadas. Formado por representantes políticos, asegura el consenso municipal y organiza y dirige los grupos de trabajo y las comisiones en las que se reúnen a personas competentes de cada municipio y en cada una de las áreas que se han definido, un total de seis, con campos tan diversos como la educación o el turismo.

Para la puesta en marcha de acciones de promoción cuenta con la colaboración de Adebisa. La Agencia de Desarrollo del Bidasoa (Adebisa-Bidasoa Activa, 1993) se crea en junio de 1992 a iniciativa de los ayuntamientos de Irun y Hondarribia y con el refrendo del Departamento de Economía de la Diputación Foral de Gipuzkoa. El trabajo de la sociedad, extensible a multitud de campos, se concentra actualmente 
en cinco departamentos distintos, además de la coordinación del Consorcio transfonterizo que, aunque teóricamente independiente del devenir de la sociedad, su actividad es dirigida desde la misma. Estos departamentos son los siguientes: Empleo, Orientación y Formación; Prospección y Formación Continua; Economía, Estudios e Innovación; Autoempleo y Centro de Empresas; y Turismo, coincidiendo con las habitualmente trabajadas en las agencias de desarrollo españolas (Campos, Sanchís y Cantero, 2002).

\section{Las acciones que impulsan relaciones sociales avanzan mientras otros campos estratégicos siguen sin abordarse}

Profundizando en las peculiaridades de cada campo abordado, se analizan las memorias anuales que publica Adebisa y que recogen también la actividad del Consorcio. En ellas observamos que ni las acciones emprendidas ni los campos trabajados presentan variaciones importantes en su andadura. ${ }^{7}$

Tomando como punto de partida la información publicada, a través de la entrevista a diferentes agentes (técnicos del Consorcios, Bidasoa Activa y representantes políticos con voz en ambas agencias), se realiza un ejercicio de valoración sobre el alcance de las diferentes acciones que en la colaboración transfronteriza se han emprendido, de las que recogemos las siguientes conclusiones.

En la última década el Consorcio apenas ha abordado nuevos campos pero sí ha reforzado el trabajo desarrollado los años anteriores multiplicando el número de iniciativas en ámbitos como el turismo, la cultura, el deporte, entre otros. En general, se resalta la posibilidad que una conurbación transfronteriza tiene a la hora de promocionarse en dos regiones y Estados diferentes.

En turismo se confirma, como principal objetivo de trabajo, la consolidación del destino Bidasoa como nueva referencia vacacional, principalmente en los sectores de los Estados francés y español. A dicho efecto, se ha configurado una serie de objetivos que se han venido reforzando a raíz de las experiencias adquiridas desde la colaboración entre las oficinas de turismo comarcales. Estos consisten en la edición conjunta de herramientas turísticas sobre la bahía, actuaciones promocionales del Destino Bidasoa y la realización de campañas publicitarias para la captación de turistas en temporada baja. También se repercute en el tejido turístico comarcal para lograr asentar de forma definitiva la importancia de la cooperación y la complementariedad turística fomentada por la Comisión de Turismo del Consorcio. Los resultados son innegables y hoy en día el trasiego de turistas de un lado a otro de la frontera se realiza con toda normalidad, obteniendo toda la comarca beneficio de este hecho.

A nivel cultural, por ejemplo, la actividad da continuidad a los certámenes iniciados años antes. El grupo de trabajo de cultura es uno de los primeros que empezó a funcionar incluso sin estar el Consorcio constituido como tal, impulsando en 1997

\footnotetext{
${ }^{7}$ En la página web del Consorcio www.bidasoa-txingudi.com se informa ampliamente sobre las actuaciones que en cada campo ha emprendido el Consorcio recientemente. Para profundizar en su evolución es necesario consultar los anuarios que Adebisa ha ido progresivamente publicando y en los que el Consorcio aparece como una de las áreas trabajadas por ellos. Esta información puede ser consultada en el enlace que se recoge a continuación: http://www.bidasoa-activa.com/es/sala-de-prensa/publicaciones. Sus conclusiones son recogidas y resumidas en Alberdi (2013).
} 
el primer "día de Txingudi", siendo esta fiesta el inicio de numerosas iniciativas impulsadas desde este grupo. El principal avance que observan se centra en la difusión que tienen ahora estos eventos. Al ser organizados por asociaciones locales que se relacionan en ámbitos regionales y nacionales diferentes, los contactos y el público que cada uno atrae es diverso y, como consecuencia, se enriquece enormemente este tipo de actos.

El proyecto de revalorización del patrimonio de Bidasoa-Txingudi da continuidad a la filosofía inicial. Se sigue desarrollando el camino que une a las tres ciudades, dando cuenta de un continuo físico y creando un itinerario que muestre a los ciudadanos los recursos comunes, compartidos y complementarios que conforman la Bahía de Txingudi. Como resultado de la reflexión iniciada y las líneas de trabajo a seguir, se perfila un conjunto de acciones, cuyo objetivo consiste en hacer avanzar las tres entidades locales de manera concreta hacia un acercamiento y una colaboración duradera, implicando directamente a los residentes de la zona, haciéndoles descubrir la cara desconocida de los atractivos del territorio (historia y medio ambiente) (Figura 2).

Figura 2: Ejemplos de ámbitos trabajados por el Consorcio Bidasoa-Txingudi

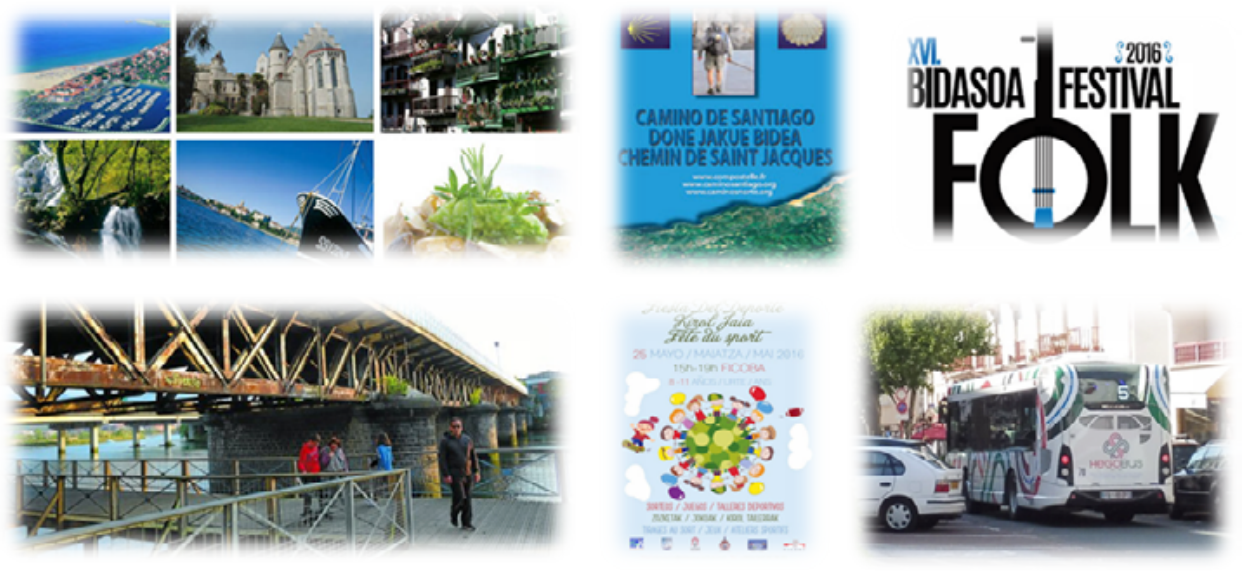

Fuente: http://www.irunhondarribiahendaye.com. Elaboración propia.

Fuera de los ámbitos trabajados los técnicos reconocen que los resultados son escasos. Inicialmente el Consorcio nace para promocionar áreas de desarrollo económico, a lo largo de su andadura se plantea asumir servicios públicos como la recogida de residuos sólidos o el abastecimiento de agua pero, generalmente por trabas administrativas, nunca llegan a materializarse. Son pocas las acciones iniciadas en estas áreas, con resultados que generalmente no superan la fase de proyecto y que rápidamente son aparcados. ${ }^{8}$

\footnotetext{
${ }^{8}$ Realidad que contrasta con afirmaciones más optimistas, como las que realizaba Rubiralta (1997), que señalaba que "en la medida que las estructuras de Estado-nación, principales responsables de la reducción del espacio pirenaico a una función secundaria y marginalizada como mera barrera fronteriza, se van disolviendo y se está poniendo fin, al mismo tiempo, a una etapa aciaga para el devenir de los Pirineos que se ha agudizado de forma especial en los últimos cien años, las perspectivas en dirección hacia una más intensa colaboración intrapirenaica van haciéndose realidad y, con ello, la unidad del mundo pirenaico puede volver a aflorar en toda su dimensión histórica".
} 
Como indicador del tipo de propuestas que han prosperado y de las que no lo han hecho, señalan la propia evolución de los diferentes planes estratégicos de la conurbación. El primero de ellos, con el nombre de Plan Estratégico del Bidasoa, se aprobó el año 1993. Se plantea como un plan económico surgido de la iniciativa de los ayuntamientos de Irun, Hondarrabia y Hendaya, con la colaboración de la Diputación Foral de Gipuzkoa, Gobierno Vasco, Consejo General de los Pirineos Atlánticos y Consejo Regional de Aquitania.

Dentro del listado de propuestas realizadas en 1993 coexistían proyectos en curso con meros esbozos de ideas que requerían de un tratamiento específico. Entre aquellas que se ejecutaron cabe destacar la realización de un centro integral de mercancías (Zaisa) inserta en la red europea de distribución, la promoción de un patrimonio público de suelo industrial con la creación de una sociedad pública gestora, la creación de un centro comercial con proyección supracomarcal o el desarrollo de puertos deportivos en localidades como Hendaya y Hondarribia. Otras sin embargo todavía hoy están en proceso de discusión, como es la construcción de un centro intermodal de mercancías. Pero lo que es realmente destacable es el número reducido de iniciativas concebidas para servir a las tres localidades.

El Plan Estratégico que se inicia el año 2000, aún vigente, a diferencia del realizado una década antes, deja de priorizar las actividades económicas para centrarse en las funciones sociales, culturales, turísticas o deportivas. Hoy en día el Consorcio carece de un nuevo plan estratégico, siendo muchas las voces que reclaman una mayor operatividad ${ }^{9}$ y que muestran la dificultad que tienen los ayuntamientos para poner en marcha iniciativas que respondan a las necesidades que una conurbación tiene en materia de transporte, servicios públicos, medio ambiente, etc. Mientras tanto esta colaboración se reduce a promover acciones que ayudan a mantener cierta relación social, es decir, actividades culturales y deportivas.

\section{Retos y oportunidades de la colaboración transfronteriza: El Consorcio ante la necesaria adaptación a las nuevas realidades institucionales}

La integración administrativa de la conurbación no avanza. Son otras las entidades que asumen gran parte del papel que debía desarrollar la figura transfronteriza. Este es el caso de la Communauté Sud Pays-Basque, ${ }^{10}$ creada el año 2006. Varios servicios, como la gestión de residuos, que se planteaban de manera colectiva en la conurbación, han pasado a manos de la Communauté, limitándose los campos de trabajo comunitario a un lado y otro de la frontera o, en el mejor de los casos, quedando a expensas de acuerdos ya no entre tres sino entre doce ayuntamientos ${ }^{11}$ (Figura 3).

\footnotetext{
${ }^{9}$ Sallaberry (2012), presidente del Consorcio y alcalde de Hendaya afirma que: "En el Consorcio hay un planteamiento común, pero la realidad es que las leyes son diferentes. No son cosas de vida o muerte, pero te queda un sabor amargo cuando intentas resolver un problema transfronterizo y no puedes porque te enredas en cuestiones jurídicas de competencias. Queda mucho por hacer para que esto vaya tan fluido como debiera".

${ }^{10}$ La Comunidad de Comunas (Communauté de Communes) es una estructura administrativa de Francia. Se trata de una entidad pública de cooperación intercomunal que ejerce, en lugar de las comunas miembros, un cierto número de competencias definidas por la ley del 6 de febrero de 1992 relativa a la administración territorial de la República francesa, modificada por la ley del 12 de julio de 1999, y posteriormente por la ley del 27 de febrero de 2002.

11 Para profundizar en las acciones que durante el periodo 2005-2012 ha realizado la Communauté consultar la publicación "D'une Communauté de Comunes à une Agglomération", recogida en la web de la entidad. Además del informe, en la misma web recoge de manera individual los objetivos, actuaciones y planes de cada una de las áreas en las que tiene competencias. También recoge informaciones más recientes, al modo de anuarios, que pueden consultarse en: www.agglo-sudpaysbasque.fr/nos-publications.html
} 
Figura 3: Aglomeración País Vasco Sur, entre Hendaya y San Juan de Luz

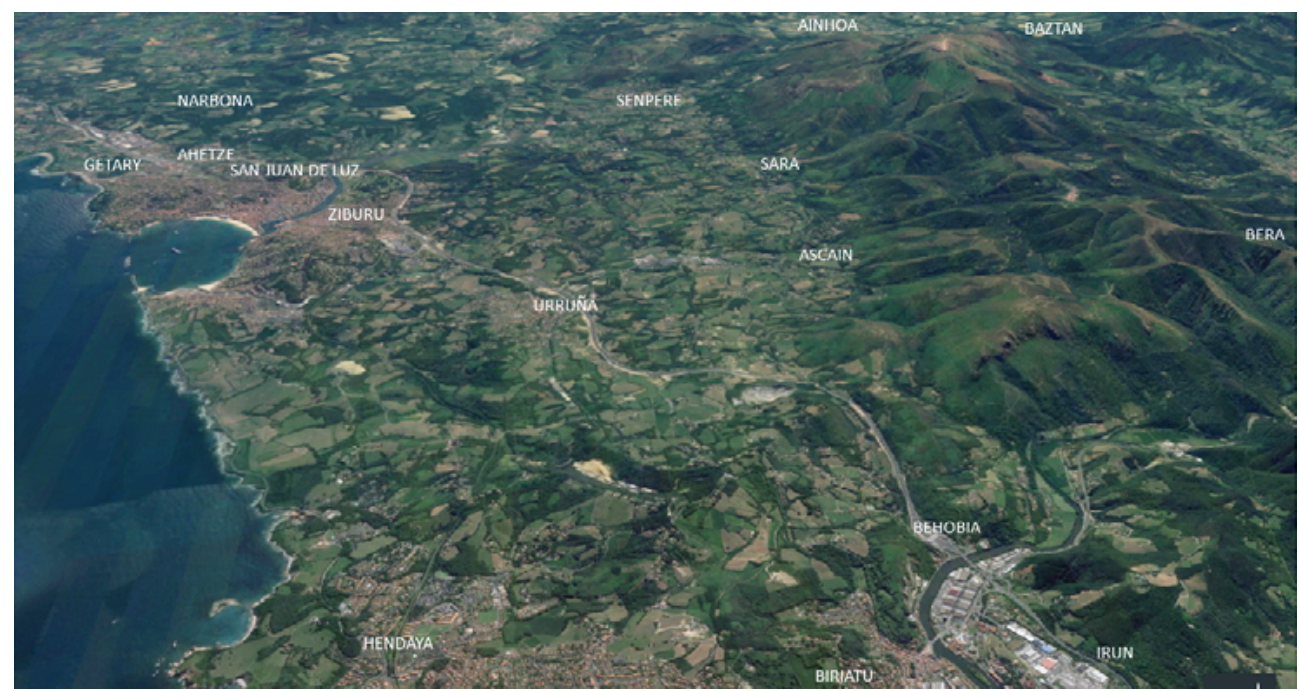

Fuente: Google Earth. Elaboración propia.

La Communauté Sud Pays Basque cuenta con una población de 64.863 habitantes (ver Tabla 2). La mitad de la población vive entre Hendaya y San Juan de Luz y otra cuarta parte en la prolongación de estos municipios en Ziburu y Urruña. El resto lo constituyen localidades más discretas, antaño rurales, salpicadas de caseríos dispersos, y actualmente sometidas a los movimientos pendulares de población que trabaja en el medio urbano y que reside en este hábitat disperso. ${ }^{12}$ A partir del año 2013 y tras superar Hendaya los 15.000 habitantes, la Communauté adquiere la subcategoría de Agglomération, aspecto que no cambia su dinámica anterior pero que le va a llevar a adquirir nuevas competencias que aún no había desarrollado, algunas de ellas de manera obligatoria. Mantenimiento de infraestructuras, transporte urbano, desarrollo de espacios públicos, etc., están entre las nuevas competencias que la Agglomération ha comenzado a gestionar.

Tabla 2: Población y empleo en la AgglomérationSud PaysBasque (2016)

\begin{tabular}{|l|r|}
\hline Población total (habitantes) & 63.014 \\
\hline Tasa de paro $(\%)$ & 10,7 \\
\hline Número de desempleados & 2.970 \\
\hline Número de empleos & 21.538 \\
\hline Empleo sector industrial (\%) & 11,9 \\
\hline Empleo sector construcción (\%) & 7,6 \\
\hline Empleo sector servicios $(\%)$ & 78,4 \\
\hline
\end{tabular}

Fuente: Bidasoa Activa (2019).

${ }^{12}$ Observatorio del Bidaso en: www.bidasoa-sudpaysbasque.com 
Además, los ayuntamientos deciden que sea la Agglomération la que aborde las relaciones transfronterizas. Aunque sea Hendaya la localidad en la que estas tienen mayor presencia, son muchos los municipios limítrofes, como Biariatu, Urruña, Sara, Senpere o Ainhoa, marcando buena parte de la muga del Bidasoa, ya con localidades navarras como Bera o Baztan. Se suceden multitud de proyectos entre localidades de un lado y otro de la frontera, algunos relacionados con saneamiento comunitario, otros con el control de la calidad de las aguas y la promoción de espacios naturales, entre otros.

Uno de ellos, InnovMugabe, está dirigido a promocionar la relación entre empresas de ambos lados de la frontera. Tras un acuerdo entre Bidasoa Activa y la Agglomération se da inicio a partir del año 2010 a un proyecto de colaboración entre empresas, una iniciativa que trata de romper con el tradicional recelo entre la población de un lado y otro del Bidasoa (Álvarez, 2010). La entrevista a sus responsables permite valorar el alcance y las posiblidades del mismo.

InnovMugabe es un proyecto de desarrollo empresarial que se mantiene activo por el apoyo recibido en diferentes convocatorias y que, a través de colaboraciones entre empresas, universidades y centros tecnológicos del Bidasoa y del Sud Pays Basque, quiere trabajar e impulsar proyectos conjuntos en torno a materias que promuevan y faciliten procesos de innovación. Los resultados no han sido espectaculares, el número de empresas que ha participado en proyectos de colaboración entre uno y otro lado no es destacable pero las relaciones iniciadas han sido positivas y se han conseguido avances, tal y como apuntan los entrevistados. ${ }^{13}$

Es más, según indican los responsables del proyecto en las entrevistas, la colaboración en materia de desarrollo económico va a continuar, independientemente de que cuente o no con ayuda económica, ya que las entidades responsables han puesto las bases para que las herramientas que se han realizado sean renovadas continuamente por los propios usuarios (catálogo de empresas, GIS, entre otras) y se han comprometido a mantener una dinámica de colaboración continuada.

De hecho, si antes de la crisis el trasvase de población de un lado a otro de la muga era evidente, ahora son las empresas del Bidasoa y de toda Gipuzkoa las que buscan ampliar su mercado en el País Vasco francés. Formar, asesorar y dirigir este interés es competencia de Bidasoa Activa y de la Agglomération Sud Pays Basque, y por ello esta colaboración es cada vez más demandada.

Pero en este desarrollo hay una entidad que pierde peso, el Consorcio Transfronterizo. Se aleja aún más la posibilidad de que la conurbación del Bidasoa adquiera peso administrativo mientras las relaciones entre entidades supramunicipales se incrementa.

\footnotetext{
${ }^{13}$ En línea con el programa europea POCTEFA (Programa Operativo de Cooperación Territorial entre Francia, España y Andorra) el proyecto se aborda desde la perspectiva de la mejora competitiva del tejido empresarial a partir del aprovechamiento y la potenciación de las oportunidades derivadas de su condición transfronteriza. Las acciones van dirigidas a empresas de todos los sectores, situadas principalmente en los polígonos empresariales de las comarcas del Bidasoa y de Sud Pays Basque. Cuenta con dos socios participantes, Bidasoa Activa y la Communauté de Communes du Sud Pays Basque y dispone de una web oficial del proyecto: www.bidasoa-sudpaysbasque.com
} 


\section{Discusión de los resultados: El Bidasoa, ante la dificultad de superar la barrera administrativa de la frontera}

La valoración de los agentes implicados en el devenir de las relaciones transfronterizas en la comarca de Txingudi-Bidasoa, junto a la reflexión crítica que se obtiene tras el análisis de más de dos décadas de noticias de hemeroteca, evidencia la pervivencia de una frontera mental, social e institucional en el Bidasoa, a pesar de que la desaparición de la barrera física. La permeabilidad de la frontera es una realidad incuestionable pero su existencia continúa aún presente y se visualiza en diferentes áreas.

- Escasa funcionalidad del Consorcio Transfronterizo. Todos los entrevistados, pero especialmente los responsables políticos resaltan las dificultades de coordinación interadministrativa como responsable del escaso avance que en materia de colaboración internacional se está produciendo. La dificultad inicial parece establecerse en el retraso producido en el carácter centralista de la administración francesa. Cualquier acción en materia de educación, promoción económica, movilidad,... ha de contar con el aval nacional y esta labor no está contemplada en la legislación vigente, quedando a menudo apartadas. ${ }^{14}$

- Excesiva administración competencial que rodea a la Bahía. El hecho internacional, el ser un área costera, la existencia de un aeropuerto internacional, el contar con un humedal protegido, un parque natural y un doble corredor dentro de la red natura 2000, el paso fronterizo... lleva a que cualquier proyecto transfronterizo se tope con la necesidad de superar mil y una barreras administrativas y que, muchas veces por imposibilidad y otras por cansancio, acabe desestimándose.

- Nuevas estructuras competenciales que debilitan las existentes. Especialmente señalado desde los representantes políticos franceses, pero reconocido por todos los entrevistados, se apunta que mientras el Consorcio ha de sortear la legislación de dos Estados diferentes, surgen nuevas estructuras administrativas que asumen competencias que inicialmente se pensaba que podían ser abordadas desde la entidad transfronteriza. A partir del año 2002 Francia posibilita crear grupos de comunas para abordar la consecución de determinadas competencias de una manera más eficaz. Con ello Hendaya aborda estas acciones uniéndose a San Juan de Luz y a otras pequeñas comunas de la zona en vez de hacerlo, dadas las dificultades a las que se había enfrentado, con Irun y Hondarribia.

- Falta de presupuestopropio para iniciar acciones transfronterizas. Los problemas económicos de las administraciones en general y, especialmente de las españolas, se ha traslucido en una reducción drástica del presupuesto desde

\footnotetext{
${ }^{14}$ Todo indica que el marco legal establecido no es eficiente y que es necesario crear un marco europeo que preserve esta colaboración de las trabas administrativas estatales. Es una reivindicación clásica, recogida por aquellas personas implicadas directamente en el progreso de estas relaciones. Llimona I Balcells (2001) ya apuntaba que hasta el momento, este desarrollo se ha producido en un contexto jurídico poco definido. La dimensión que ha adquirido esta cooperación exige un marco jurídico estable no solo a nivel estatal, sino principalmente, tal como viene reclamándose por parte de los diferentes actores, a nivel europeo.
} 
el año 2009. Con ello, las posibilidades de actuación del Consorcio se han reducido aún más, encontrándose en este momento en una situación crítica y quedando en gran medida a merced de la captación de proyectos financiados desde la Unión Europea.

- Pervivencia de la frontera como barrera mental, social y económica. A pesar de los años de colaboración y de la permeabilidad residencial, la frontera mental entre los habitantes de ambos lados continúa. Los habitantes buscan soluciones en su territorio, rehúyen la relación y el idioma limita las posibilidades de comunicación. Apenas hay avances en este campo y las deficiencias, especialmente en el lado español, son constatables. Tampoco hay una permeabilidad del tejido empresarial. El asociacionismo apenas fructifica y cada empresa busca desarrollar su actividad en su territorio respectivo. ${ }^{15}$

A pesar de las deficiencias observadas la relación transfronteriza sigue avanzando. El continuo urbano al que nos enfrentamos sigue creciendo, la conciencia sobre la necesidad de impulsar la relación es evidente entre las instituciones locales y ello se manifiesta en diferentes campos:

- Son muchas las personas, empresas y entidades que observan cómo las posibilidades de negocio se incrementan, especialmente la hostelería. La apertura de una conexión marítima entre Hendaya y Hondarribia, con una cifra que superó los trescientos mil viajeros el año 2017, beneficia claramente al sector servicios de ambos municipios, especialmente a Hondarrabia, con una economía volcada en las posibilidades que la restauración le aporta.

- La promoción, tanto como destino turístico como de cualquier evento que se celebre, se beneficia del trabajo en red. Toda difusión realizada es internacional y cualquier evento se propaga a la Eurociudad Donostia-Bayona, a las regiones de Aquitania y País Vasco y a los Estados francés y español.

- La estructura técnica y política ya está generada. La colaboración entre los municipios es continua y fluida, la apuesta por potenciar estas relaciones no es cuestionada y son muchos los proyectos que de manera conjunta ya se han materializado. Es más, los mismos municipios tratan de sortear las dificultades administrativas diseñando, cada uno desde su territorio y competencia, proyectos dirigidos al conjunto de la ciudadanía del Bidasoa: paseos por la bahía, carril bici, transporte público comunitario, etc.

Y todo ello ocurre en un momento clave para la colaboración a ambos lados del Bidasoa. La crisis de la construcción en España provocó que el que diera el salto ya no solo fuera el empresario con cierto volumen de negocio sino que incluso el autónomo comenzara a ofrecer su servicio, y así continúa una década después. Este fenómeno

\footnotetext{
${ }^{15}$ Los nuevos consorcios que vienen creándose a lo largo de la presente década nacen con unos objetivos y perspectivas amplios que, si atenemos al ejemplo del Bidasoa, previsiblemente verán frenadas sus pretensiones. Sirva como ejemplo las aportaciones que Manero realiza respecto al Consorcio del Duero, siendo consciente que "la satisfacción de los fines que la animan no será una tarea fácil ni cómoda, pues son aún numerosas las incógnitas que plantea la gestión y que sólo la aplicación práctica de la experiencia, con sus señales correctoras, permitirá resolver al compás de las modificaciones reglamentarias que puntualmente habrán de llevarse a cabo a fin de asegurar los niveles de eficacia pretendidos" (Manero, 2012, p. 270).
} 
supera el ámbito de Hendaya y llega hasta Biarritz y Bayona. La situación actual obliga al tejido empresarial a moverse rápido. El particular busca soluciones inmediatas y si la administración no responde simplemente no acude a ella, tal y como señalan los responsables del consorcio.

\section{A modo de conclusión: Una colaboración que avanza más lenta de lo esperado}

El objeto de la investigación, el análisis del Consorcio Transfronterizo del Bidasoa como un ejemplo de las transformaciones que las fronteras internas están sufriendo en la UE, hacía presagiar un escenario en el que la separación de dos comunidades provocada por la frontera podía estar en claro proceso de superación.

El apoyo de los programas europeos o la existencia de un espacio con relaciones simétricas y de proximidad contribuía a considerar un estadio en el que la colaboración socioeconómica y la cohesión social se vieran profundamente desarrolladas.

Estudios más centrados en la tipología de modalidad elegida, la figura del consorcio, mostraban el alcance que podían tener estas relaciones y, especialmente en aquellos casos en los que la masa poblacional fuera significativa, abarcando incluso la planificación común de determinados servicios públicos o la colaboración económica en pos de lograr un mayor valor añadido de determinados productos.

El espacio en estudio, la conurbación de la bahía de Txingudi, entorno próximo, reducido, delimitado físicamente y con claros nexos económicos, culturales, lingüísticos y sociales presagiaba a priori el avance rápido de las relaciones intercomunitarias. La rapidez de los avances institucionales, la firma del tratado de Bayona con el objeto de dar cobertura a las relaciones intercomunitarias como la planteada en el Bidasoa, e incluso la reciente ubicación de la oficina de coordinación de la Eurogión EuskadiNueva Aquitania-Navarra en Hendaya podían presagiar un escenario de amplia superación del hecho fronterizo.

Los resultados de la investigación, sin embargo, dejan en evidencia que las expectativas esperadas no se han cumplido. Las declaraciones realizadas por representantes políticos locales a lo largo de la década actual junto a las indicaciones de los entrevistados confirman que son las acciones culturales, deportivas y promocionales las que avanzan. La puesta en marcha de iniciativas comunitarias en materia de servicios y equipamientos, tan importantes para una conurbación, no han sido abordadas, y Hendaya ha de aliarse con otras comunas con las que, a pesar de ubicarse en un radio no superior a los $30 \mathrm{~km}$, no comparte vecindad. Otro tanto ocurre con la planificación urbanística y la ordenación territorial, imposible de ser abordada en documentos conjuntos.

La justificación de la situación presentada no estriba precisamente en la falta de interés de los tres municipios por abordar estos servicios de manera conjunta, de la que indudablemente obtendrían beneficios económicos y sociales (hospital comarcal, enseñanza trilingüe en español, francés y euskera, servicios unificados de transporte público con acceso a las conexiones regionales e incluso nacionales de cada localidad...). En el estudio se llega a la conclusión de que son las trabas administrativas, con ritmos totalmente diferentes e incluso incompatibles, las que imposibilitan que estos campos puedan ser abordados. 
A la rémora competencial se le une la pervivencia aún de una frontera mental o psicológica y, aunque ya en menor medida, lingüística. Cada comunidad ve a la otra con reticencia, apenas se establecen relaciones y todo ello afecta en el proceder económico que, lejos de aprovechar sinergias, busca su medio de vida de manera independiente una de la otra.

A pesar de que la conurbación Vasca sea un espacio geo-social con innegables nexos culturales, políticos, sociales y económicos que deberían de facilitar la colaboración transfronteriza, se topa con muchas de las barreras que ya se apuntaban en otros espacios fronterizos. En cierta medida, la situación reflejada parece ser habitual entre los consorcios transfronterizos europeos, en los que, por ritmos administrativos o por la pervivencia de barreras psicológicas, la colaboración que realmente se establece es menor que la deseada. Pero a diferencia de otros ejemplos, esta no se produce por falta de reflexión, por situaciones económicas coyunturales, por una excesiva dependencia de fondos económicos europeos o por falta de coordinación con otras escalas de colaboración institucional. En el caso vasco la dificultad de conjugar competencias entre un Estado autonómico con las de uno centralizado.

Diluir el borde fronterizo parece, por tanto, un proceso lento y sinuoso afrontable solo si cambian condiciones administrativas y socioeconómicas. Precisamente en este campo se están produciendo las principales novedades. La crisis inmobiliaria española, con el consecuente incremento de las tasas de paro, ha llevado a que una parte del tejido empresarial y autónomo centrado en la construcción mire por primera vez al País Vasco francés como lugar donde trabajar. Los movimientos laborales se incrementan, al igual que los comerciales y turísticos, pero la administración no ha sabido adaptarse a las necesidades que esta superación de la barrera conlleva.

En este proceso Bidasoa-Txingudi ha de buscar aliados. La Agglomération Sud Pays Basque (2013) se puede convertir en una pieza clave para aumentar la relación entre ambos lados, ampliándola en un volumen poblacional más equilibrado entre un lado y el otro. Sin descuidar aspectos dotacionales, la colaboración entre ambas entidades es una oportunidad para ampliar los campos de actuación tradicionales del Consorcio y hacer que la colaboración empresarial sea definitivamente uno de sus pilares de desarrollo. A su vez, la Agglomération ha asumido la responsabilidad de algunos de los campos que el Consorcio tradicionalmente había trabajado, como cultura o turismo.

Todo indica que avanzamos en las relaciones transfronterizas del Bidasoa de la conurbación de tres municipios al entendimiento entre Adebisa y la Agglomération. La nueva área geográfica permite abordar actuaciones en campos que en las dos décadas de relación entre un lado y otro del Bidasoa no se habían materializado. El proyecto InnovMugabe y el interés mostrado por el empresario parece augurar un interesante campo de desarrollo.

Es ahora labor del Consorcio y de sus aliados, la Agencia de Desarrollo del Bidasoa y la Aglomeración Sud Pays Basque, que este interés sea algo más que una relación coyuntural, apoyando y formando al empresario, intensificando las relaciones entre agentes económicos, abarcando nuevos campos e impulsando un interés bidireccional. 


\section{Agradecimientos}

Este artículo se deriva del proyecto "La cooperación transfronteriza en Europa. Una geopolítica a escala local", Programa Estatal de I+D+I orientada a los retos de la sociedad (código CSO2015-67124-R).

\section{Referencias}

Adebisa-Bidasoa Activa. (1993). Informe anual. Publicación anual Irun. Recuperado de http://www.bidasoa-activa.com/es/sala-de-prensa/publicaciones

Agglomération Sud Pays Basque (2013). D’une Communauté de Comunes à une Agglomération. Recuperado de www.agglo-sudpaysbasque.fr

Alberdi, J. C. (2006). La cooperación municipal en el Bidasoa: de la acción local al consorcio internacional. Lurralde: Investigación y Espacio, 29, 87-113.

Alberdi, J. C. (2013). Evolución de las relaciones transfronterizas en El Bidasoa: de la conurbación a la communauté. Geographicalia, 63, 5-26.

Alexeev, M. (Ed.) (1999). Center-Periphery Conflict in Post-Soviet Russia: A Federation Imperiled. Nueva York: St. Martin's Press.

Altemir, A. B. (1989). La cooperación transfronteriza en Europa: especial referencia al marco pirenaico. Revista CIDOB d'afers Internacionals, 17, 37-54.

Álvarez, G. (2010). Primera reunión de balance de actividades del proyecto transfronterizo InnovMugabe liderado por Bidasoa activa. Diario Digital de la Comarca del Bidasoa. Irun. Recuperado de www.bidasoaldia.com

Anderson, J. y O'Dowd, L. (1999). Borders, border regions and territoriality: contradictory meanings, changing significance. Regional Studies Association, 33, 593-604.

Arbaiza, J. K. (2006). Proyectos de cooperación transfronteriza en el País Vasco: objetivos y realidad, Lurralde, 29, 143-177.

Barrios, M. D. L. (2013). Re-significando la frontera: el caso de la Eurociudad Chaves-Verín. Boletín de la Asociación de Geógrafos Españoles, 61, 309-328.

Berger, F. (2005). Développement de l'emploi transfrontalier auLuxembourg et portrait sociodémographique des frontaliers. Luxembourg: CEPS/Instead

Bidasoa Activa. (2019). El territorio en cifras. Recuperado de https://www.bidasoa-sudpaysbasque.com/es/herramientas-consulta/territorio-cifras

Campos, V., Sanchís, J. R. y Cantero, S. (2002). Un estudio sobre el perfil y las características del agente de desarrollo local en la Comunidad Valenciana. Análisis comparativo con el resto de España. RVEH, 5, 147-182.

Feliu, J., Berzi, M., Rufí, J. V., Castañer, M. y Llussà, R. (2013). Análisis de los proyectos y actores transfronterizos España-Francia en el período 2007-2013. Geographicalia, 63, 64, 75-93.

Fernández, C. (1985). La frontera hispano francesa y las relaciones de vecindad (especial referencia al sector fronterizo del País Vasco). Bilbao: Servicio Editorial Universidad del País Vasco/EuskalHerrikoUnibertsitatea.

Fourny, M.-Ch. y Vélasco-Graciet, H. (2005). L'eurorégion Aquitaine Euskadi Navarre, un espace transfrontalier émergent?, Sud-Ouest Européen, 19, 71-84. 
Galdos, R. y Ruiz, E. (1995). Potencialidades y proyectos de cooperación transfronteriza en el País Vasco. En Actos del VII Coloquio Ibérico de Geografía. Cáceres.

Guerrero, L. M. (2001). La entrevista en el método cualitativo. Facultad de Ciencias Sociales, Universidad de Chile. Recuperado de http://rehue. csociales. uchile. cl/ investigacion/genetica/cg04. Htm

Harguindéguy, J. B y Rodríguez, P. (2010). Gobernanza multi-nivel y política regional europea. La implementación de INTERREg III-A España-Francia. Revista de Estudios Regionales, 88, 199-222.

Heredero, M. I. y Olmedillas, B. (2009). Las fronteras españolas en Europa: de INTERREG a la cooperación territorial europea. Investigaciones Regionales, 16, 191-215.

Ibarra, P. e Ikardo, I. (1997). Movimientos sociales transfronterizos en la bahía de Txingudi. La construcción del espacio vasco-aquitano (pp. 115-128). Lejona: Servicio Editorial de la Universidad del País Vasco.

Koff, H. (2008). La política fronteriza comparada y las estructuras del poder. Estudios Políticos, 32, 195-226

Kupiszewski, M. (2004). Consequences of EU enlargement for freedom of movement between the Council of Europe member states. Europa, 21(11), 45-53.

Kuus, M. (2010). Critical geopolitics. En R. Denemark (Ed.), The International Studies Encyclopedia. Vol. II (pp. 683-701). Chichester: Wiley-Blackwell.

Llimona I Balcells, J. (2001). La cooperación transfronteriza en la frontera pirenaica: un largo camino hacia su consolidación. Tempo Exterior, 3.

Lois, R. C. y Carballo, A. (2015). La frontera hispano-lusa en la actualidad: una visión geográfica. Revista de Historiografía, 23, 191-214.

Lozano, P. J. (2007). La Eurociudad Baiona-Donostia. ¿Ejemplo o utopía en la colaboración transfronteriza?. Boletín de la Asociación de Geógrafos Españoles, 44, 325-340.

Manero, M. F. (2012). La cooperación intermunicipal como estrategia de ordenación y desarrollo territorial en espacios transfronterizos: la Agrupación Europea de Cooperación Territorial Duero-Douro. Boletín de la Asociación de Geógrafos Españoles, 58, 249-272.

Márquez, J. A. (Ed.). (2016). Desarrollo en la frontera del Bajo Guadiana: Documentos para la cooperación Luso-Andaluza (Vol. 172). Huelva: Servicio de Publicaciones de la Universidad de Huelva.

Martín-Uceda, J. y Castañer, M. (2018). Actores y proyectos transfronterizos en las fronteras ibéricas: análisis comparativo a partir de Interreg IV-A. Boletín de la Asociación de Geógrafos Españoles, 78, 154-179. doi: http://dx.doi.org/10.21138/ bage. 2432

Matthiessen, C. W. (2005). The Öresund Area: Pre-and post-bridge cross-border functional integration: the bi-national regional question. Geo Journal, 61, 31-39.

O'Dowd, L. (2010). From a 'borderless world' to a 'world of borders': bringing history back in. Environment and Planning D: Society and Space, 28, 1031-1050.

Oliveras, X. (2013). La cooperación transfronteriza en la Cerdanya (frontera España-Francia). Boletín de la Asociación de Geógrafos Españoles, 62, 25-48.

Oliveras-González, X., Colomb, C. y Durà-Guimerà, A., (2016). La cooperación transfronteriza en el contexto pirenaico: retos de la multiplicidad. En J. M. Trillo e I. Pires (Eds.), Fronteras en la investigación peninsular: temáticas y enfoques contemporáneos/Fronteiras na investigação peninsular: temáticas e abordagems contemporâneas. 
Santiago de Compostela: Servicio de Publicacións e Intercambio Cientítifico da Universidade de Santiago de Compostela.

Perkmann, M. y Sum, N. (Eds.). (2002). Globalization, Regionalization, and Cross-Border Regions. Houndsmills: Palgrave.

Prokkola, E. (2008). Making bridges, removing barriers. Cross-border cooperation and identity at the Finnish-Swedish border. Oulu: Nordia Geographical Publications.

Rubiralta, F. (1997). El espacio pirenaico y la construcción europea: Fundamento histórico y revitalización de un área transfronteriza. En La construcción del espacio vasco-aquitano (pp. 25-56). Lejona: Servicio Editorial de la Universidad del País Vasco.

Rogut, A. y Welter, F. (2012). Cross-border cooperation within an enlarged Europe: Görlitz/Zgorzelec. En D. Smallbone, F. Welter y M. Xheneti (Eds.), Cross-border entrepreneurship and economic development in Europe's border regions (pp. 67-88). Cheltenham: Edward Elgar.

Sallaberry, J. B. (2012). Lo transfronterizo no ha ido tan rápido como debía y ahora lo estamos pagando. En Morondo (corresponsal) Diario Digital de Gipuzkoa. Donostia. Recuperado de www.diariovasco.com

Sansinenea, G. (2001). La Eurociudad Bayona-San Sebastián. Lurralde, 24, 45-76.

Scott, J. (2007). Cross-border regionalization in an enlarging EU.Hungarian-Austrian and German-Polish cases. En H. Koff (Ed.). Deceiving (dis)appearances: analyzing current developments in Europeanand North American border regions (pp. 37-58). Bruselas: P.I.E. Peter Lang.

Sohn, C., Reitel, B. y Walther, O. (2009). Cross-border metropolitan integration in Europe: the case of Luxembourg, Basel and Geneva. Environment and Planning: Government and Policy, 27, 922-939.

Statec. (2007). Le Commerce transfrontelier du Luxembourg dans la Grande Région. Economie et Statistiques, 18, 1-13.

Ventura-Fernández, J. (2013). Redes para la cooperación a distintas escalas: Asociacionismo municipal, regiones europeas y programas transnacionales. Revista de Estudios Andaluces, 30, 48-77.

Woodward, K. y Jones, J. P. (2005). On the border with Deleuze and Guattari. En H. Van Houtum et al. (Eds.), Bordering Space (234-248). Aldershot, Ashgate.

Juan Cruz Alberdi Collantes

Español. Licenciado en Geografía por la Universidad de Zaragoza, doctorado por la Universidad del País Vasco, donde ejerce actualmente como profesor titular. Su actividad profesional e investigadora queda ligada a su participación en los diferentes programas de desarrollo rural que se van desarrollando en el País Vasco. Su aportación principal radica en el tipo de geografía que realiza, es decir, una geografía participativa y aplicada en proyectos que se están materializando y en cuya ejecución toma parte. Líneas de investigación: ordenación y desarrollo de los espacios rurales. Publicación reciente: Alberdi, J. C. (2019). El paisaje atlántico vasco ante la marginalización ganadera: claves para su conservación. Boletín de la Asociación de Geógrafos Españoles, (80), 6 . 